\title{
Induction of Lipin1 by ROS-Dependent SREBP-2 Activation
}

\author{
Kyuhwa Seo and Sang Mi Shin \\ College of Pharmacy, Chosun University, Gwangju, Korea
}

\begin{abstract}
Lipin1 was identified as a phosphatidate phosphatase enzyme, and it plays a key role in lipid metabolism. Since free radicals contribute to metabolic diseases in the liver, this study investigated the effects of free radicals on the regulation of Lipin1 expression in Huh7 and AML12 cells. Hydrogen peroxide induced mRNA and protein expression of Lipin1 in Huh7 cells, which was assayed by quantitative RT-PCR and immunoblotting, respectively. Induction of Lipin1 by hydrogen peroxide was confirmed in AML12 cells. Hydrogen peroxide treatment significantly increased expression of sterol regulatory element-binding protein (SREBP)-2, but not SREBP-1. Moreover, nuclear translocation of SREBP-2 was detected after hydrogen peroxide treatment. Hydrogen peroxide-induced Lipin1 or SREBP-2 expression was significantly reduced by $\mathrm{N}$-acetyl-L-cysteine treatment, indicating that reactive oxygen species (ROS) were implicated in Lipin1 expression. Next, we investigated whether the hypoxic environments that cause endogenous ROS production in mitochondria in metabolic diseases affect the expression of Lipin1. Exposure to hypoxia also increased Lipin1 expression. In contrast, pretreatment with antioxidants attenuated hypoxia-induced Lipin1 expression. Collectively, our results show that ROS activate SREBP-2, which induces Lipin1 expression.
\end{abstract}

Key words: Lipin1, Reactive oxygen species, SREBP, Oxidative stress, Liver

\section{INTRODUCTION}

The liver is an important organ for metabolism of xenobiotics and nutrients (1). Through this process, the liver is often exposed to reactive oxygen species (ROS), which are a metabolic byproducts $(1,2)$. In metabolic diseases such as dibetes and obesity, liver dysfunction is often associated with high levels of ROS (2-4). Increased levels of free radicals impair mitochondrial function and interfere with hepatic energy metabolism (2). Although the liver has a variety of antioxidant systems, it has been reported that oxidative

\footnotetext{
Correspondence to: Sang Mi Shin, College of Pharmacy, Chosun University, 309 Pilmun-daero, Dong-gu, Gwangju 61452, Korea E-mail: smshin@chosun.ac.kr
}

Abbreviations: DAG, diacylglycerol; fld, fatty liver dystrophy; NAC, $\mathrm{N}$-acetyl-L-cysteine; Nrf1, NF-E2-related factor 1; PAP, phosphatidate phosphatase; PGC- $1 \alpha$, PPAR $\gamma$ coactivator- $1 \alpha$; PPAR, peroxisome-proliferator-activated receptor; ROS, reactive oxygen species; SREBP, sterol regulatory element-binding protein; VLDL, very-low-density lipoprotein.

This is an Open-Access article distributed under the terms of the Creative Commons Attribution Non-Commercial License (http:// creativecommons.org/licenses/by-nc/3.0) which permits unrestricted non-commercial use, distribution, and reproduction in any medium, provided the original work is properly cited. stress plays an important role in the remission of liver disease (2). Thus, controlling the levels of ROS is an important way to prevent liver damage from metabolic disorders.

Lipin1 is involved in the synthesis of triglyceride, the main energy storage material in the body $(5,6)$. The substrate required for the synthesis of triglycerides is diacylglycerol (DAG), which is produced by phosphatidate phosphatase (PAP) $(5,6)$. Lipin1 is an enzyme that displays PAP activity $(5,7)$. It binds phosphatidic acid and converts it to DAG when fatty acid levels are increased (5). Lipin1 has been reported to regulate lipid metabolism in many tissues, including fat, muscles, and testes (8-10). Moreover, Lipin1 possesses a nuclear localization signal, a signal to promote transcriptional activity, and the PAP enzyme active site necessary for lipid synthesis $(5,6)$. Indeed, as a transcriptional coactivator or corepressor, Lipin1 interacts with transcription factors and nuclear receptors associated with lipid metabolism $(11,12)$. This suggests that Lipin1 is more than just an enzyme involved in simple lipid synthesis, and it may be involved in other types of intracellular metabolic signaling.

Several studies have reported that Lipin1 regulates the synthesis of triglycerides, in addition to lipoprotein metabolism in liver $(5,6,13,14)$. The Lipin1 gene was first identified with a deficiency mutation in the fatty liver dystrophy (fld) mice $(10,15)$. The fld mice with a Lipinl deficiency 
display hypertriglycemia, insulin resistance, and neonatal fatty liver $(10,15)$. Similarly, the expression of Lipin1 was significantly reduced in HIV patients with lipodystrophy (16). In addition, hepatic very-low-density lipoprotein (VLDL) secretion was significantly increased in fld mice $(11,13)$. Moreover, Lipin1 interacts with nuclear receptors, including peroxisome-proliferator-activated receptor (PPAR) $\alpha, \operatorname{PPAR} \gamma, \operatorname{PPAR} \gamma$ coactivator-1 $\alpha$ (PGC-1 $\alpha$ ), hepatocyte nuclear factor $4 \alpha$, and glucocorticoid receptor, and it regulates their transcriptional activity (11-13). This suggests that Lipin1 plays an important role in lipid homeostasis, even though Lipin1 expression level in liver is relatively low compared to that in fat and muscle. However, the mechanism of Lipin1 regulation by ROS, which play an important role in the pathology of liver disease, is still poorly studied.

Given the importance of Lipin1 regulation in hepatic metabolic diseases, we investigated the toxicological contribution of ROS in the regulation of Lipin1. In particular, we studied the effect of endogenous or exogenous increases in ROS levels on Lipin1 expression in hepatocyte-derived cells.

\section{MATERIALS AND METHODS}

Materials. Antibodies against Lipin1, sterol regulatory element-binding protein (SREBP)-1, SREBP-2, and $\beta$-tubulin were purchased from Santa Cruz Biotechnology (Santa Cruz, CA, USA). Anti-Lamin antibody was obtained from Cell Signaling Technology (Danvers, MA, USA). Anti- $\beta$ actin antibody, hydrogen peroxide, $N$-acetyl-L-cysteine (NAC), trolox, and other reagents were supplied by Sigma (St. Louis, MO, USA).

Cell culture. Mouse hepatocellular AML12 cell lines were obtained from the American Type Culture Collection (Manassas, VA, USA) and maintained in a 1:1 mixture of DMEM and Ham's F12 medium. Additionally, AML12 cells were cultured in media supplemented with $0.005 \mathrm{mg} /$ $\mathrm{mL}$ insulin, $0.005 \mathrm{mg} / \mathrm{mL}$ transferrin, $5 \mathrm{ng} / \mathrm{mL}$ selenium, and $40 \mathrm{ng} / \mathrm{mL}$ dexamethasone. Huh7 cells were maintained in RPMI-1640 and all cells were maintained in $10 \%$ fetal bovine serum (FBS), $50 \mathrm{U} / \mathrm{mL}$ penicillin, and $50 \mu \mathrm{g} / \mathrm{mL}$ streptomycin at $37^{\circ} \mathrm{C}$ under $5 \% \mathrm{CO}_{2}$ in a humidified incubator. All cells were cultured in 6-well plates for $2 \sim 3$ days to reach $70 \sim 80 \%$ confluency and then they were serumstarved overnight before experimental treatment. For the hypoxic treatments, cells were exposed to $94 \% \mathrm{~N}_{2}, 5 \% \mathrm{CO}_{2}$, and $1 \% \mathrm{O}_{2}$ at $37^{\circ} \mathrm{C}$ in a hypoxia-inducing incubator (Galaxy 48R, New Brunswick [Eppendorf], Hamburg, Germany).

Immunoblot analysis. Preparation of whole-cell lysates, subcellular fractionation, and immunoblot analysis were performed as previously described (17). Briefly, the lysates were resolved by SDS-PAGE, and electrophoretically blot- ted onto nitrocellulose membranes. The membranes were incubated with primary antibodies overnight. The membranes were then exposed to horseradish peroxidase-conjugated secondary antibodies. Protein bands were identified by the ECL chemiluminescence system (GE Healthcare, Buckinghamshire, UK). Equal loading of proteins was verified by immunoblotting for $\beta$-actin, $\beta$-tubulin or Lamin.

RNA isolation and RT-PCR analysis. Total RNA from Huh7 cells was extracted using a Trizol reagent (Invitrogen, Carlsbad, CA, USA). Next, cDNAs were generated using oligo $(\mathrm{dT})_{16}$ primers and the RT Premix (Bioneer, Daejeon, Korea), and PCR products were visualized on a 2\% agarose gel stained with EtBr. Data were normalized to $G A P D H$ expression. PCR was performed using primers specific for genes encoding human Lipin1 (5'-CCCGACCTTCAACACCTAAAAGT-3' [forward] and 5'-TGGACTCTTTCATCTTGTGTGGA-3' [reverse]), Lipin2 (5'-AAGAAGTGGCAGGTGATGC- $3^{\prime}$ [forward] and 5'-CAGCTTTCCAAACCGAACG-3' [reverse]), and GAPDH (5'-GAAGATGGTGATGGGATTTC-3' [forward] and 5'-GAAGGTGAAGGTCGGAGTC-3' [reverse]).

Measurement of mitochondrial ROS. The production of mitochondria-derived superoxide was examined using the mitoSOX red reagent (Invitrogen). MitoSOX is a fluorogenic dye for selective detection of mitochondrial superoxide in live cells (18). The cells were cultured in 12-well plates, and mitoSOX was added directly to the medium at a final concentration of $5 \mu \mathrm{M}$. The cells were then incubated at $37^{\circ} \mathrm{C}$ for $0.5 \mathrm{hr}$, and then detached using trypsin-EDTA. Cells were pelleted and suspended in PBS supplemented with $1 \%$ FBS. Stained cells were quantified using a fluorescence microplate reader (Gemini XPS, Molecular Devices, Sunnyvale, CA, USA). Production of mitochondrial ROS was normalized to the protein concentration and calculated relative to the vehicle-treated control using the following formula: ROS production (fold of control) $=[$ (relative fluorescence unit of treated sample)/(protein concentration of treated sample) $] /[$ (relative fluorescence unit of vehicletreated control $) /($ protein concentration of vehicle-treated control)].

Statistical analysis. Values are expressed as the means \pm SE. Statistical significance was calculated by analysis of variance (ANOVA). Differences were considered significant at $p<0.05$ or $p<0.01$.

\section{RESULTS}

Induction of Lipin1 by ROS. To investigate the regulation of Lipin1 expression by ROS, Huh7 cells were treated with $500 \mu \mathrm{M}$ hydrogen peroxide. The expression of Lipin1 was increased in a time-dependent manner in the presence 

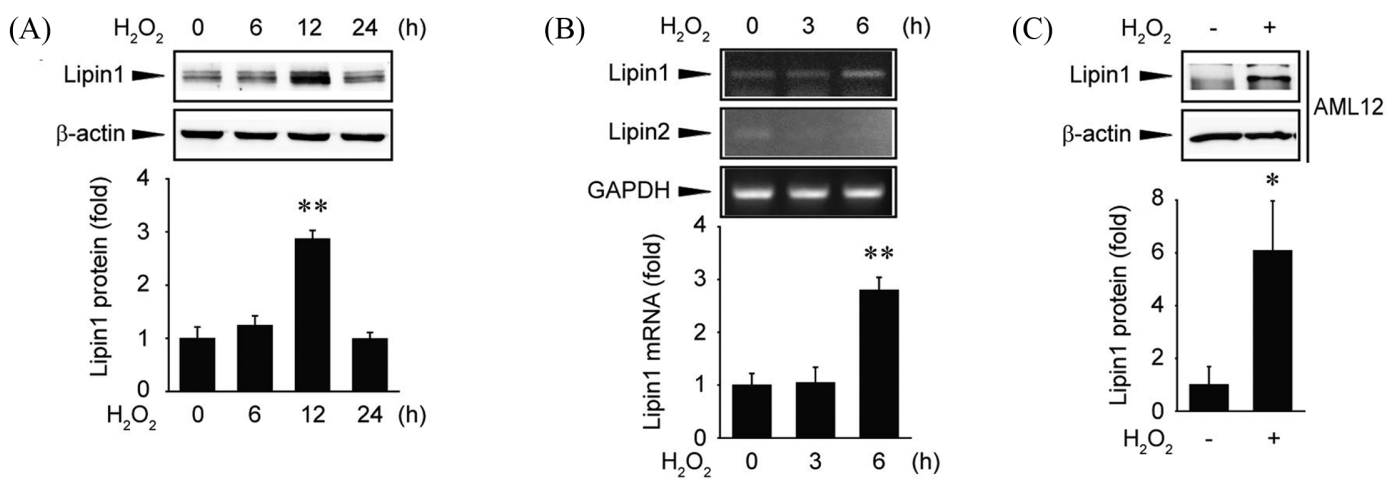

Fig. 1. Upregulation of Lipin1 expression by hydrogen peroxide. (A) Immunoblot analysis. Huh7 cells were treated with hydrogen peroxide $(500 \mu \mathrm{M})$ for $0 \sim 24 \mathrm{hr}$. The blots shown are representative of data from at least three different replicates; ${ }^{* *} p<0.01$ compared to the control. (B) RT-PCR assays. Huh7 cells were treated with hydrogen peroxide $(500 \mu \mathrm{M})$ for $0 \sim 6 \mathrm{hr}$. Lipin expression was analyzed by RT-PCR. The results shown are representative of data from at least three different replicates; ${ }^{* *} p<0.01$ compared to the control. (C) Immunoblot analysis. AML12 cells were treated with hydrogen peroxide $(500 \mu \mathrm{M})$ for $24 \mathrm{hr}$. The blots shown are representative of data from at least three different replicates; ${ }^{*} p<0.05$ compared to the control.

of ROS (Fig. 1A). Next, we quantified Lipin1 mRNA abundance to determine whether the changes in Lipin1 protein expression resulted from changes in its mRNA levels. Huh7 cells treated with hydrogen peroxide showed an increase in Lipin1 mRNA levels at $6 \mathrm{hr}$ (Fig. 1B). However, treatment with hydrogen peroxide did not affect the mRNA abundance of Lipin2 (Fig. 1B). This suggests that the increase in Lipin1 protein expression involves an increase in its mRNA expression. Additional experiments were performed in AML12 cells to determine whether the increase in Lipin1 expression following exposure to free radicals was also observed in other hepatocyte-derived cell lines. Interestingly, increased Lipin1 protein expression was also observed in AML12 cells after hydrogen peroxide treatment (Fig. 1C). Collectively, these results clearly show that Lipin1 is upregulated by ROS.
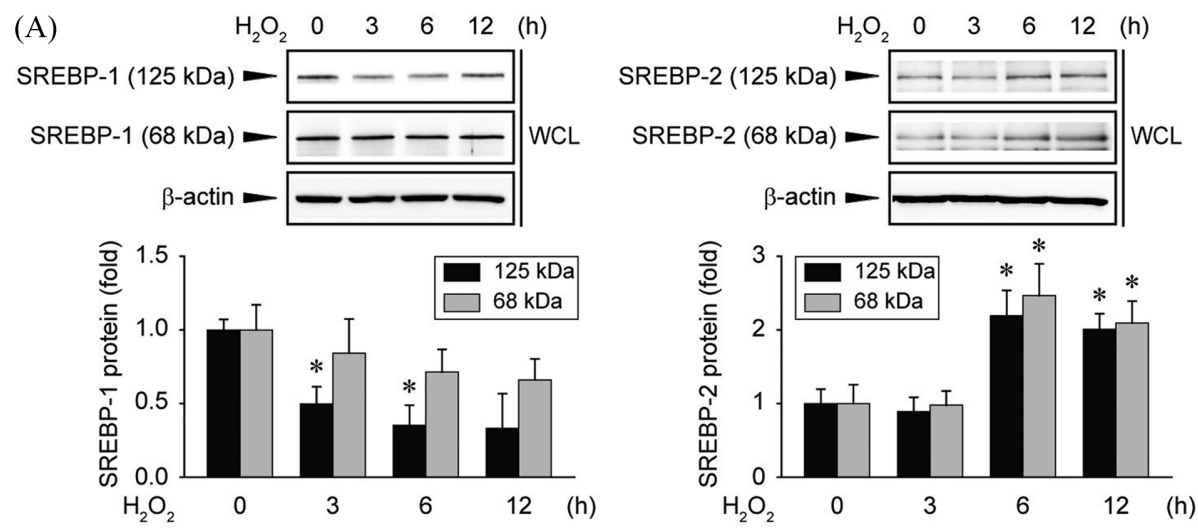

(B)

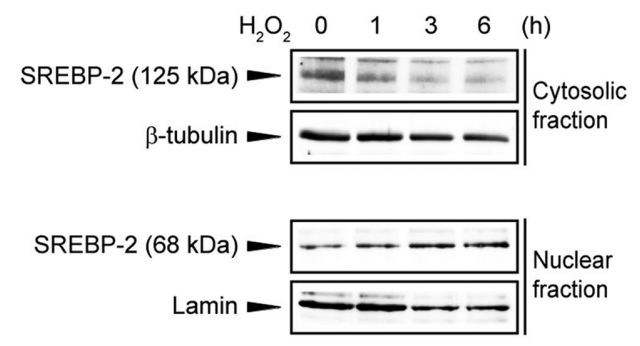

Fig. 2. Effects of hydrogen peroxide on SREBP regulation. (A) Immunoblot analysis. Huh7 cells were treated with hydrogen peroxide $(500 \mu \mathrm{M})$ for $0 \sim 12 \mathrm{hr}$. The blots shown are representative of data from at least three different replicates; ${ }^{*} p<0.05$ compared to the control; WCL, whole cell lysates. (B) Immunoblot analysis. SREBP-2 was immunoblotted using cytosolic or nuclear extracts from Huh7 cells treated with hydrogen peroxide $(500 \mu \mathrm{M})$ for $0 \sim 6 \mathrm{hr}$. The blots shown are representative of data from at least three different replicates. 
Involvement of SREBP in Lipin1 induction by ROS. Next, we investigated which transcription factor is involved in the induction of Lipin1. SREBP is a transcription factor involved in regulating various lipid synthesis genes in the liver, including Lipin1 $(5,19)$. Huh7 cells were treated with hydrogen peroxide and the expression levels of SREBP-1 and SREBP-2 were determined. In the case of SREBP-1, the level of the full-length isoform was significantly reduced following hydrogen peroxide treatment, but no change was observed for the abundance of the cleaved isoform (Fig. $2 \mathrm{~A}$, left). On the other hand, the levels of both full-length and cleaved forms of SREBP-2 were increased after hydrogen peroxide treatment (Fig. 2A, right). Next, the intracellular dynamics of SREBP-2 were examined. Expression of the full-length SREBP-2 was decreased in the cytoplasm after hydrogen peroxide treatment, and the cleaved form was accumulated in the nucleus (Fig. 2B). Taken together, the increase in expression of Lipin1 following oxidative stress induced by treatment with hydrogen peroxide may be due to an increase in the expression and activity of SREBP-2.

Inhibition of Lipin 1 expression by antioxidants. Next, we examined whether antioxidants can inhibit the expression of Lipin1 following hydrogen peroxide treatment. Huh7 cells were pretreated with NAC and exposed to hydrogen peroxide. The treatment with NAC significantly decreased the protein levels of Lipin1 following hydrogen peroxide exposure (Fig. 3A). The expression of SREBP-2 was also quantified following treatment with NAC. It was found that NAC treatment significantly reduced the expression of
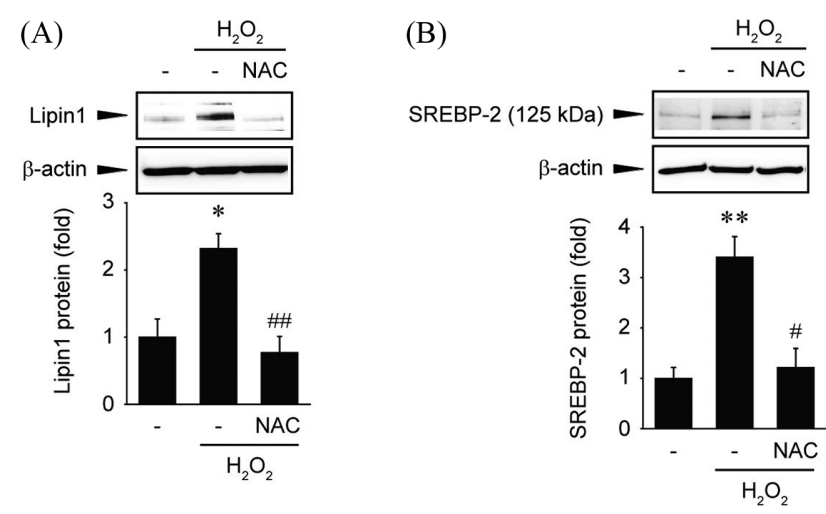

Fig. 3. Effects of ROS on Lipin1 regulation. (A) Immunoblot analysis. Huh7 cells were pretreated with NAC $(10 \mathrm{mM})$ and continuously incubated with hydrogen peroxide $(500 \mu \mathrm{M})$ for $12 \mathrm{hr}$. The blots shown are representative of data from at least three different replicates; ${ }^{*} p<0.05$ compared to vehicle-treated cells, ${ }^{\#} p<0.01$ compared to hydrogen peroxide-treated cells. (B) Immunoblot analysis. Huh7 cells were pretreated with NAC $(10 \mathrm{mM})$ and continuously incubated with hydrogen peroxide $(500 \mu \mathrm{M})$ for $6 \mathrm{hr}$. The blots shown are representative of data from at least three different replicates; ${ }^{* *} p<0.01$ compared to vehicle-treated cells, ${ }^{*} p<0.05$ compared to hydrogen peroxide-treated cells.
SREBP-2 (Fig. 3B). These results indicate that Lipin1 may be regulated by free radicals and ROS-dependent SREBP-2 expression.

The effect of hyoxia-induced ROS production in Lipin 1 induction. Next, we investigated whether the expression of Lipin1 is regulated by endogenous free radicals. In a hypoxic environment, the use of oxygen in the mitochondria is limited (20). Thus, the amount of ROS is increased (20). First, we tried to detect cytosolic ROS level by using dichlorofluorescin in hypoxia. However, hypoxia did not significantly affect dichlorofluorescin fluorescence intensity when compared with normoxia-exposed cells (data not shown). Therefore, we conducted experiment for measurement of mitochondrial ROS. When cells were cultured in a hypoxic environment, the amount of ROS originating from the mitochondria was increased (Fig. 4A). Next, the effect of increased ROS levels on Lipin1 expression was examined. Cells were cultured for $24 \mathrm{hr}$ in a hypoxic environment, and Lipin1 expression was increased (Fig. 4B). In addition, pretreatment with antioxidants, such as NAC (5 $\mathrm{mM})$ or trolox $(100 \mu \mathrm{M})$, decreased Lipin1 expression (Fig. 4B). Exposure to hypoxic condition for $24 \mathrm{hr}$ caused severe damage to cells. Therefore, we used NAC at a lower con-
(A)

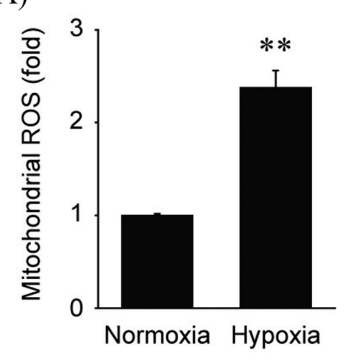

(B)

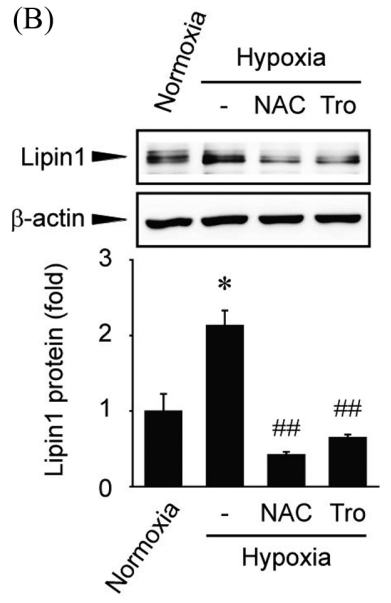

Fig. 4. Effect of mitochondrial ROS on Lipin1 induction following hypoxia. (A) Measurement of mitochondrial ROS production. Huh7 cells were incubated under normoxic $\left(21 \% \mathrm{O}_{2}\right)$ or hypoxic $\left(1 \% \mathrm{O}_{2}\right)$ conditions for $6 \mathrm{hr}$. After staining with mitoSOX, the cells were harvested. Mitochondrial ROS production was determined using a fluorescence reader. Data are expressed as the means $\pm \mathrm{SE}$ from at least three independent experiments; ${ }^{* *} p<0.01$ compared to the control. (B) Immunoblot analysis. Huh7 cells were pretreated with NAC $(5 \mathrm{mM})$ and trolox (Tro, $100 \mu \mathrm{M})$, and then incubated under hypoxic conditions $\left(1 \% \mathrm{O}_{2}\right)$ for $24 \mathrm{hr}$. The blots shown are representative of data from at least three different replicates; ${ }^{*} p<0.05$ compared to the normoxia-incubated cells, ${ }^{\#} p<0.01$ compared to the hypoxia-incubated cells. 
centration under hypoxia. In general, many in vitro studies including our results, $2 \sim 10 \mathrm{mM}$ concentrations of NAC were used in hepatocytes (18,21-23). Hence, $5 \mathrm{mM}$ NAC is considered to be a proper concentration for scavenging ROS production. These results suggested that stress-induced ROS upregulate Lipin1 expression.

\section{DISCUSSION}

This study showed that Lipin1 was induced by endogenous and exogenous ROS in hepatocyte-derived cell lines. The increase in expression of Lipin1 protein was accompanied by a corresponding increase in mRNA levels. Moreover, it was found that the transcription factor SREBP-2 might contribute to ROS-dependent Lipin1 induction. These results suggest a mechanism in which oxidative stress causes lipid accumulation in the liver.

It has been reported that expression of Lipin1 is increased under stress conditions $(11,14)$. Glucocorticoid, a stress hormone, increases hepatic PAP activity and the synthesis of triglycerides $(24,25)$. In addition, partial hepatectomy, diabetes, and alcohol consumption have also been reported to increase PAP activity in the liver (26-29). Our results indicated that oxidative stress induces Lipin1, which supports the possibility that various stress conditions could increase Lipin1 levels as a result of excessive ROS production. Hirotsu et al., reported that the antioxidant response element is present in the promoter region of Lipin1, and NFE2-related factor 1 (Nrf1) binds this region to regulate its transcription (30). However, the precise signaling pathway mediated by Nrf1 that controls Lipin1 induction remains to be elucidated.

SREBPs are transcription factors that regulate the expression of genes involved in lipid metabolism, including Lipin1 $(5,19)$. After a two-step proteolytic cleavage, SREBPs are competent to transactivate genes involved in lipid synthesis (19,31). Sterol depletion and ethanol exposure have been reported to increase SREBP-dependent Lipin1 expression (32,33). Of the two isoforms of SREBP, studies have principally focused on the regulation of SREBP-1 and its induction of Lipin1 expression (5,32,33). In breast cancer cells, hydrogen peroxide induces SREBP-1 expression, and ultimately increases FAS levels (34). However, the present study shows that SREBP-2 might be involved in the regulation of Lipin1 expression during oxidative stress. SREBP-2 is relatively specific for the regulation of cholesterol homeostasis rather than triglyceride levels (19). In relation to cholesterol regulation, Lipin1 has been reported to have dual functions $(11,13,35)$. It can either increase or decrease VLDL secretion from the liver $(11,13,35)$. In the fld mouse model, loss of Lipin1 expression increases plasma VLDL levels $(11,13)$. Meanwhile, hepatic VLDL secretion is increased in cells that overexpress Lipin1 $(13,35)$. To understand the effects of ROS on Lipin1-mediated cholesterol homeostasis, further studies how it modulates SREBP-2 expression and activation is required.

In conclusion, our results showed that ROS induce Lipin 1 by activating SREBP-2. This research provides useful insights into the mechanisms of lipotoxicity under oxidative stress in the liver.

\section{ACKNOWLEDGMENTS}

This research was supported by Basic Science Research Program through the National Research Foundation of Korea (NRF) funded by the Ministry of Education (Grant Number: NRF-2016R1D1A1B03934436).

Received April 25, 2017; Revised May 30, 2017; Accepted June 12, 2017

\section{REFERENCES}

1. Liska, D.J. (1998) The detoxification enzyme systems. Altern. Med. Rev., 3, 187-198.

2. Cichoz-Lach, H. and Michalak, A. (2014) Oxidative stress as a crucial factor in liver diseases. World J. Gastroenterol., 20, 8082-8091.

3. Fernandez-Sanchez, A., Madrigal-Santillan, E., Bautista, M., Esquivel-Soto, J., Morales-Gonzalez, A., Esquivel-Chirino, C., Durante-Montiel, I., Sanchez-Rivera, G., Valadez-Vega, C. and Morales-Gonzalez, J.A. (2011) Inflammation, oxidative stress, and obesity. Int. J. Mol. Sci., 12, 3117-3132.

4. West, I.C. (2000) Radicals and oxidative stress in diabetes. Diabet. Med., 17, 171-180.

5. Chen, Y., Rui, B.B., Tang, L.Y. and Hu, C.M. (2015) Lipin family proteins-key regulators in lipid metabolism. Ann. Nutr. Metab., 66, 10-18.

6. Reue, K. and Dwyer, J.R. (2009) Lipin proteins and metabolic homeostasis. J. Lipid Res., 50, S109-S114.

7. Reue, K. and Brindley, D.N. (2008) Thematic review series: Glycerolipids. Multiple roles for lipins/phosphatidate phosphatase enzymes in lipid metabolism. J. Lipid Res., 49, 24932503.

8. Donkor, J., Zhang, P., Wong, S., O’Loughlin, L., Dewald, J., Kok, B.P., Brindley, D.N. and Reue, K. (2009) A conserved serine residue is required for the phosphatidate phosphatase activity but not the transcriptional coactivator functions of Lipin-1 and Lipin-2. J. Biol. Chem., 284, 29968-29978.

9. Donkor, J., Sariahmetoglu, M., Dewald, J., Brindley, D.N. and Reue, K. (2007) Three mammalian lipins act as phosphatidate phosphatases with distinct tissue expression patterns. J. Biol. Chem., 282, 3450-3457.

10. Peterfy, M., Phan, J., Xu, P. and Reue, K. (2001) Lipodystrophy in the fld mouse results from mutation of a new gene encoding a nuclear protein, lipin. Nat. Genet., 27, 121-124.

11. Finck, B.N., Gropler, M.C., Chen, Z., Leone, T.C., Croce, M.A., Harris, T.E., Lawrence, J.C., Jr. and Kelly, D.P. (2006) Lipin 1 is an inducible amplifier of the hepatic PGC-1 $\alpha$ / PPAR $\alpha$ regulatory pathway. Cell Metab., 4, 199-210.

12. Kim, H.E., Bae, E., Jeong, D.Y., Kim, M.J., Jin, W.J., Park, 
S.W., Han, G.S., Carman, G.M., Koh, E. and Kim, K.S. (2013) Lipin1 regulates PPAR $\gamma$ transcriptional activity. Biochem. J., 453, 49-60.

13. Bou Khalil, M., Blais, A., Figeys, D. and Yao, Z. (2010) Lipin The bridge between hepatic glycerolipid biosynthesis and lipoprotein metabolism. Biochim. Biophys. Acta, 1801, 12491259.

14. Mylonis, I., Sembongi, H., Befani, C., Liakos, P., Siniossoglou, S. and Simos, G. (2012) Hypoxia causes triglyceride accumulation by HIF-1-mediated stimulation of lipin 1 expression. J. Cell Sci., 125, 3485-3493.

15. Langner, C.A., Birkenmeier, E.H., Ben-Zeev, O., Schotz, M.C., Sweet, H.O., Davisson, M.T. and Gordon, J.I. (1989) The fatty liver dystrophy $(f l d)$ mutation. A new mutant mouse with a developmental abnormality in triglyceride metabolism and associated tissue-specific defects in lipoprotein lipase and hepatic lipase activities. J. Biol. Chem., 264, 7994-8003.

16. Lindegaard, B., Larsen, L.F., Hansen, A.B., Gerstoft, J., Pedersen, B.K. and Reue, K. (2007) Adipose tissue lipin expression levels distinguish HIV patients with and without lipodystrophy. Int. J. Obes. (Lond.), 31, 449-456.

17. Seo, K., Yang, J.H., Kim, S.C., Ku, S.K., Ki, S.H. and Shin, S.M. (2014) The antioxidant effects of isorhamnetin contribute to inhibit COX-2 expression in response to inflammation: a potential role of HO-1. Inflammation, 37, 712-722.

18. Shin, S.M. and Kim, S.G. (2009) Inhibition of arachidonic acid and iron-induced mitochondrial dysfunction and apoptosis by oltipraz and novel 1,2-dithiole-3-thione congeners. Mol. Pharmacol., 75, 242-253.

19. Eberle, D., Hegarty, B., Bossard, P., Ferre, P. and Foufelle, F. (2004) SREBP transcription factors: master regulators of lipid homeostasis. Biochimie, 86, 839-848.

20. Schonenberger, M.J. and Kovacs, W.J. (2015) Hypoxia signaling pathways: modulators of oxygen-related organelles. Front. Cell Dev. Biol., 3, 42.

21. Chen, Y., Liu, J.M., Xiong, X.X., Qiu, X.Y., Pan, F., Liu, D., Lan, S.J., Jin, S., Yu, S.B. and Chen, X.Q. (2015) Piperlongumine selectively kills hepatocellular carcinoma cells and preferentially inhibits their invasion via ROS-ER-MAPKs-CHOP. Oncotarget, 6, 6406-6421.

22. Hou, J., Cui, A., Song, P., Hua, H., Luo, T. and Jiang, Y. (2015) Reactive oxygen species-mediated activation of the Src-epidermal growth factor receptor-Akt signaling cascade prevents bortezomib-induced apoptosis in hepatocellular carcinoma cells. Mol. Med. Rep., 11, 712-718.

23. Kretzmann, N.A., Chiela, E., Matte, U., Marroni, N. and Marroni, C.A. (2012) N-acetylcysteine improves antitumoural response of Interferon alpha by $\mathrm{NF}-\mathrm{\kappa B}$ downregulation in liver cancer cells. Comp. Hepatol., 11, 4.

24. Pittner, R.A., Fears, R. and Brindley, D.N. (1985) Effects of cyclic AMP, glucocorticoids and insulin on the activities of phosphatidate phosphohydrolase, tyrosine aminotransferase and glycerol kinase in isolated rat hepatocytes in relation to the control of triacylglycerol synthesis and gluconeogenesis. Biochem. J., 225, 455-462.
25. Pittner, R.A., Fears, R. and Brindley, D.N. (1985) Interactions of insulin, glucagon and dexamethasone in controlling the activity of glycerol phosphate acyltransferase and the activity and subcellular distribution of phosphatidate phosphohydrolase in cultured rat hepatocytes. Biochem. J., 230, 525-534.

26. Whiting, P.H., Bowley, M., Sturton, R.G., Pritchard, P.H., Brindley, D.N. and Hawthorne, J.N. (1977) The effect of chronic diabetes, induced by streptozotocin, on the activities of some enzymes of glycerolipid synthesis in rat liver. Biochem. J., 168, 147-153.

27. Brindley, D.N., Cooling, J., Glenny, H.P., Burditt, S.L. and McKechnie, I.S. (1981) Effects of chronic modification of dietary fat and carbohydrate on the insulin, corticosterone and metabolic responses of rats fed acutely with glucose, fructose or ethanol. Biochem. J., 200, 275-283.

28. Sturton, R.G., Pritchard, P.H., Han, L.Y. and Brindley, D.N. (1978) The involvement of phosphatidate phosphohydrolase and phospholipase A activities in the control of hepatic glycerolipid synthesis. Effects of acute feeding with glucose, fructose, sorbitol, glycerol and ethanol. Biochem. J., 174, 667-670.

29. Mangiapane, E.H., Lloyd-Davies, K.A. and Brindley, D.N. (1973) A study of some enzymes of glycerolipid biosynthesis in rat liver after subtotal hepatectomy. Biochem. J., 134, 103112.

30. Hirotsu, Y., Hataya, N., Katsuoka, F. and Yamamoto, M. (2012) NF-E2-related factor 1 (Nrf1) serves as a novel regulator of hepatic lipid metabolism through regulation of the Lipin1 and PGC-1 $\beta$ genes. Mol. Cell. Biol., 32, 2760-2770.

31. Han, J.Y., Park, S.H., Yang, J.H., Kim, M.G., Cho, S.S., Yoon, G., Cheon, S.H. and Ki, S.H. (2014) Licochalcone suppresses LXR $\alpha$-Induced hepatic lipogenic gene expression through AMPK/Sirt1 pathway activation. Toxicol. Res., 30, 19-25.

32. Hu, M., Wang, F., Li, X., Rogers, C.Q., Liang, X., Finck, B.N., Mitra, M.S., Zhang, R., Mitchell, D.A. and You, M. (2012) Regulation of hepatic Lipin-1 by ethanol: role of AMP-activated protein kinase/sterol regulatory element-binding protein 1 signaling in mice. Hepatology, 55, 437-446.

33. Ishimoto, K., Nakamura, H., Tachibana, K., Yamasaki, D., Ota, A., Hirano, K., Tanaka, T., Hamakubo, T., Sakai, J., Kodama, T. and Doi, T. (2009) Sterol-mediated regulation of human Lipin 1 gene expression in hepatoblastoma cells. $J$. Biol. Chem., 284, 22195-22205.

34. Furuta, E., Pai, S.K., Zhan, R., Bandyopadhyay, S., Watabe, M., Mo, Y.Y., Hirota, S., Hosobe, S., Tsukada, T., Miura, K., Kamada, S., Saito, K., Iiizumi, M., Liu, W., Ericsson, J. and Watabe, K. (2008) Fatty acid synthase gene is up-regulated by hypoxia via activation of Akt and sterol regulatory element binding protein-1. Cancer Res., 68, 1003-1011.

35. Bou Khalil, M., Sundaram, M., Zhang, H.Y., Links, P.H., Raven, J.F., Manmontri, B., Sariahmetoglu, M., Tran, K., Reue, K., Brindley, D.N. and Yao, Z. (2009) The level and compartmentalization of phosphatidate phosphatase-1 (lipin1) control the assembly and secretion of hepatic VLDL. $J$. Lipid Res., 50, 47-58. 